\title{
Analyzing the Complex Decisions on Information Technology and Information System Outsourcing Vendor Selection by a Pair wise Comparison Approach
}

\author{
A.Megala ${ }^{\top}$, C. Veeramani ${ }^{2 *}$ \\ \{amegala84@gmail.com ${ }^{1}$, veerasworld@yahoo.com ${ }^{2}$ \} \\ Assistant Professor, Department of Mathematics, Nandha Engineering College (Autonomous), \\ Erode. ${ }^{7}$, Assistant Professor, Department of Applied Science (Mathematics), PSG college of \\ Technology, Coimbatore, India ${ }^{2}$
}

\begin{abstract}
IT outsourcing is the model of utilizing resources outside of an organization to manage some information technology activities. Organizations often outsource their activities because it is cheaper than to buy and manage their resources and infrastructure. Choose the vendor is the overhead process. Consider the multi-attribute of IT outsourcing choice. This article discusses eleven success factors in IT outsourcing that must be focused and suggests the use of the Pair wise comparison approach. The Analytical Hierarchy Process (AHP) is a mathematical problem-solving tool that has gained widespread adoption across many industries. After studying the structure of a problem, the AHP method was utilized. This study explains how this structure works. B is a method for managing and comprehending crucial decisions that is well structured.
\end{abstract}

Keywords: Please list your keywords in this section.

\section{Introduction}

Many firms are focusing on information technology outsourcing as a result of the current massive competitive and worldwide transaction infrastructure, as well as the advancement in information technologies. Information technology outsourcing has become so widespread as a result of its commercial benefits and hazards that managers can no longer avoid it. An organization's outsourcing activities play a critical part in the organization's overall operation. Any organization's product quality and pricing are determined by the excellence of its vendors. The ramifications of wrong selection become more serious. Examining, managing, and selecting providers is critical for businesses. The goal of the process is to identify vendors who are best suited to meet the organization's demands. Vendor selection decisions are challenging due to the evaluation of many aspects. The AHP technique gives a quantitative measuring of these methods and factors for comparison.

In order to solve the dilemma of selecting vendors, we suggest the AHP technique for selecting IT outsourcing suppliers. To assess the outsourcing problem, set the criteria weights, and finalize, we will apply the AHP approach. The aim is to enhance a mathematical decision model, which is assist managers in selecting the best. The study's following sections are stated as follows: the next section will provide background on vendor selection. The proposed outsourcing vendor selection approach is explained in Section 3. In section 4, you'll see an 
example of a case study from real life. Finally, in Section 5, concluding observations will be made.

\section{Literature Review}

When businesses outsource a substantial portion's operations, they must go through a vendor selection process. When there are many objectives that conflict, MCDM is utilized to address the problem. IT outsourcing is becoming a more crucial strategy for businesses nowadays. Because of the cost savings, increased profitability and services, and the benefit of global corporate competitiveness, IT outsourcing is becoming more popular (Karami \& Guo, 2012). Onshore (inside the same nation), nearshore (within a common border), and offshore outsourcing are the three types of outsourcing (countries sea border). Each company has its own set of requirements for employing IT outsourcing, which varies depending on the needs of the company. Thakur and Anbanandam (2015), for example, argue in e-banking systems is utilized to improve the system's understanding of consumer satisfaction. Companies have implemented outsourcing tactics to enhance the standard at a reduced cost despite working with insufficiently skilled human resources as a result of advancements in information and communication technologies (Faisal \& Raza, 2016). Hence, the research was done to discover criteria that could help companies make better outsourcing decisions when selecting competent vendors. Since the 1960s, academics have been examining supplier selection, with early research focused mostly on financial implications and single-criterion assessments. Price, quality, delivery time, and financial and performance measures are among the criteria devised by many academics for selecting suppliers. Multi-Criteria. Both physical and intangible (Digalwar et al. (2014) criteria are used to select suppliers in IT outsourcing MCDM. Quality, cost/price, and delivery/reliability are essential variables in the IT outsourcing choices of the banking system, according to Thakur and Anbanandam (2015).

Successful IT outsourcing was aided by selection criteria. Many of the criteria for selecting an IT outsourcing provider are based on expert experience and the demands of the firm, according to history (Qiang \& Li, 2015). Some studies, on the other hand, provided criteria for successful IT outsourcing projects. Consider Kronawitter, Wentzel, and Papadaki's (2013) study, which provided a set of criteria for successful IT outsourcing. IT outsourcing management, according to Hanlie Smuts et al. (2010), should be concerned with twelve factors that ensure IT outsourcing success. Alexandrova (2012) conducted a study that identified significant factors that influence the success of an IT outsourcing relationship. Next, Ismail and Razali (2014) proposed on shoring and offshore for software testing outsourcing, based on knowledge, language and culture, political stability, staff turnover, reputation, and appropriate resources. Ullah Khan et al. (2010) cited cost cutting, enough infrastructure, competent project management, product quality, and maintenance as criteria for offshore software development.

\section{The AHP Method}

AHP gives a method for calculating the scores and weights for the many criteria utilized in decision-making [8]. It presents a methodology for selecting outsourcing vendors based on data gathered throughout the outsourcing selection process by AHP. The AHP [10-11] is a 
technique developed by Saaty for systematically considering facts or information relevant to a decision. The AHP is typically used to assist in the resolution of choice issues that involve ambiguity and a large number of criteria. It is based on three principles: first, establish a hierarchy; second, establish priorities; and third, maintain logical coherence.

\section{- Step 1:}

A complicated problem is broken into sub-problems, with the goal of quantifying contributions to an overarching purpose or emphasis. Dickson underlined that the three most significant variables in vender evaluation were cost, quality, and delivery performance [12]. According to Weber et al., quality is the most significant consideration for selecting a vendor, which is followed by delivery performance and cost [13]. As the significance of vendor selection and global competitiveness has grown, the approach to old criteria has been revised to meet additional requirements due to the position of vendors in the distribution chain. Many other elements must be considered in modern management in order to create a long-term supplier relationship [15]. As a result, the eleven criteria - Cost / Financial Management (n1), Professional Human Resource / Staff Management (n2), Quality and Service Performance (n3), Relationship and Communication Management (n4), Knowledge (n5), Flexibility (n6), Technology / Physical Infrastructure (n7), Executive Management Level Support (n8), MultiCulture and Language (n9), Reputation (n10), and Risk Management (n11) - were chosen.

\section{- Step 2:}

The "priority" assigned to each element in the hierarchy is established by comparing pair wise the contributions of each element at a lower level in terms of the criteria for which, there is a causal link [15]. AHP uses a nine-level conventional comparison scale for multiple matches comparison. (see Table I [10] ).

Table - I. Scale of Relative Importance

\begin{tabular}{|c|l|c|l|}
\hline Intensity of importance & Definition & $\begin{array}{l}\text { Intensity } \\
\text { of importance }\end{array}$ & Definition \\
\hline 1 & Equally Significance & 2 & Weak \\
\hline 3 & Moderately Significance & 4 & Moderate + \\
\hline 5 & Strongly Significance & 6 & Strong + \\
\hline 7 & $\begin{array}{l}\text { Very strongly or demonstratively } \\
\text { Significance }\end{array}$ & 8 & Strong ++ \\
\hline 9 & Extremely importance & & \\
\hline
\end{tabular}

The collection of criteria is defined as $\eta=\{n j \mid j=1,2 \ldots n\}$. As shown in table $I$, the result of the pair wise comparison on $n$ criteria can be summed in a ( $\mathrm{n} \times \mathrm{n})$ evaluation matrix A, where each member $a_{i j}$ is the quotient of the criteria's weights. The relative importance is determined by the right eigenvector $(w)$, which corresponds to the greatest eigenvector $\left(\lambda_{\max }\right)$ as,

$$
\mathrm{AW}=\lambda \max ^{*} \mathrm{~W}
$$

The matrix A has rank 1 and $\max =\mathrm{n}$ if it is absolutely consistent. The weights can be established by normalizing any of A's columns or rows. Each of the hierarchy's subsystems is treated with the technique described above. The degree to which the alternatives contribute to the emphasis is shown by their overall, relative priorities. These priorities are a synthesis of local priorities, as well as the result of an test process that allowed the opinions of the numerous stakeholders to be integrated ${ }^{[16]}$.

- $\quad$ Step 3: Consistency Check

It is mandatory. The consistency index (CI) is 


$$
\mathrm{CI}=\left(\lambda_{\max }-\mathrm{n}\right) /(\mathrm{n}-1)
$$

The consistency ratio (CR), which can be applied to determine whether or not the assessments are adequately consistent, is determined as the ratio of the $\mathrm{CI}$ and the random consistency index (RI), as shown in table II, as CR = CI/RI. The approved upper limit for CR is the value 0.1 . If the final consistency ratio is higher than the specified value, the evaluation method must be repeated in order to increase consistency. The consistency measurement would be used to analyze decision makers' consistency and the hierarchies' overall consistency.

Table II. Consistency Index

\begin{tabular}{|l|l|l|l|l|l|l|l|l|l|l|l|}
\hline Matrix rank & 1 & 2 & 3 & 4 & 5 & 6 & 7 & 8 & 9 & 10 & 11 \\
\hline RI & 0.00 & 0.00 & 0.58 & 0.90 & 1.12 & 1.24 & 1.32 & 1.41 & 1.45 & 1.49 & 1.51 \\
\hline
\end{tabular}

\section{A CASE STUDY}

\subsection{Background of the Business}

Praistma Technology is a company that offers business consultancy and technology management services. A routine information management system is being outsourced by the organisation. The most difficult aspect of the outsourcing programme is choosing the correct provider. The firm has three vendors to pick from (vendor $\mathrm{A}$, vendor $\mathrm{B}$, vendor $\mathrm{C}$, vendor $\mathrm{D}$, and vendor E). We employ the AHP technique to assist the firm in selecting the ideal provider.

\subsection{Problem Hierarchy}

This study we decide to use the eleven criteria based on the firm's specific situation: Cost / Financial Management (n1), Professional Human Resource / Staff Management (n2), Quality and Service Performance (n3), Relationship and Communication Management (n4), Knowledge (n5), Flexibility (n6), Technology / Physical Infrastructure (n7), Executive Management Level Support (n8), Multi-Culture and Language (n9), Reputation (n10) and Risk Management and Assessment (n11), The hierarchy diagram (Figure1) is composed for the above criteria, which are discussed in section 3.

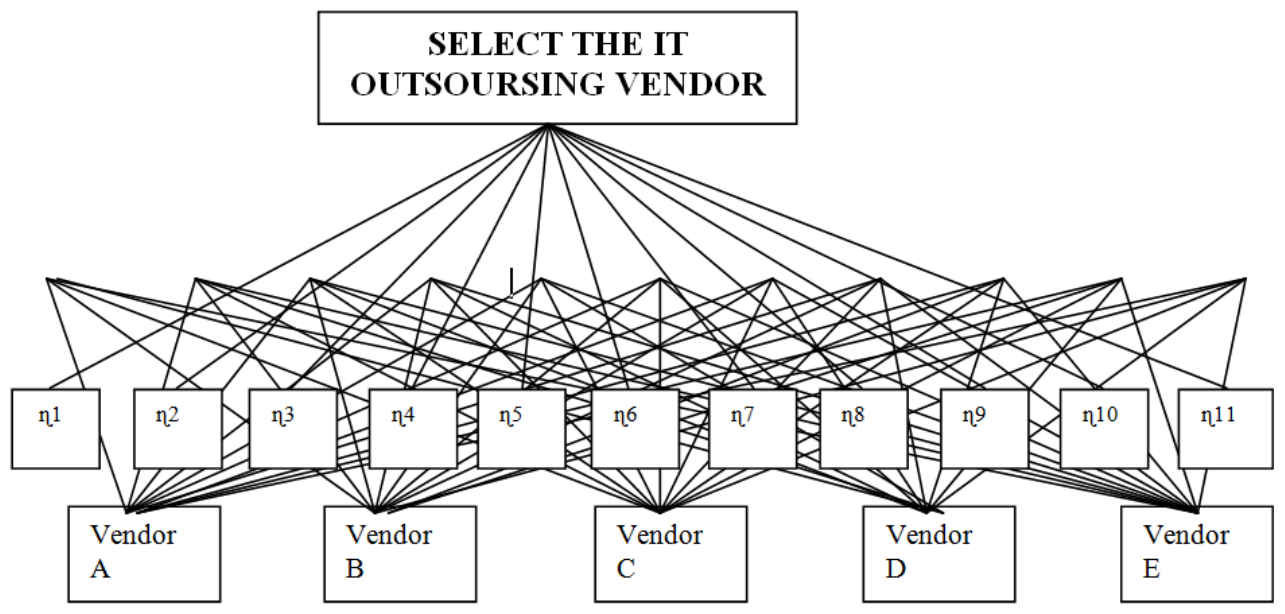

Figure 1. Problem Hierarchy 


\subsection{Criteria Weights}

Experts began comparing the factors using the computational method provided in AHP. Criteria Professional Human Resource / Staff Management ( 2 2), for example, have a lower priority than Cost / Financial Management ( $\eta 1$ ). So, according to Table - I, the importance value 3 on the satty scale was plotted in the concern cell. All of the values in the matrix are dependent on how all of the criteria are compared to one another. Following that, they were given the square matrix displayed in Table III.

Table - III. Pair wise Comparison Matrix - A

\begin{tabular}{|c|c|c|c|c|c|c|c|c|c|c|c|}
\hline $\mathbf{A}$ & $\eta \mathbf{1}$ & $\eta 2$ & $n 3$ & $n 4$ & n5 & n6 & $n 7$ & $\eta 8$ & $\eta^{9}$ & $\eta 10$ & n11 \\
\hline$\eta 1$ & 1 & 3 & $1 / 7$ & $1 / 3$ & $1 / 4$ & $1 / 3$ & $1 / 7$ & $1 / 4$ & $1 / 3$ & 6 & 7 \\
\hline$n 2$ & $1 / 3$ & 1 & $1 / 8$ & $1 / 6$ & $1 / 5$ & $1 / 3$ & $1 / 3$ & $1 / 4$ & $1 / 6$ & $1 / 5$ & $1 / 6$ \\
\hline$\eta 3$ & 7 & 8 & 1 & 7 & 6 & 7 & 5 & 7 & 7 & 7 & 7 \\
\hline$n 4$ & 3 & 6 & $1 / 7$ & 1 & 3 & 2 & $1 / 3$ & 2 & 2 & 2 & $1 / 5$ \\
\hline n5 & $1 / 5$ & 5 & $1 / 6$ & $1 / 3$ & 1 & 4 & $1 / 2$ & 5 & 5 & 3 & $1 / 4$ \\
\hline n6 & 3 & 3 & $1 / 7$ & $1 / 2$ & $1 / 4$ & 1 & $1 / 3$ & 2 & 6 & 4 & $1 / 6$ \\
\hline n7 & 7 & 3 & $1 / 5$ & 3 & 2 & 3 & 1 & 5 & 5 & 3 & $1 / 4$ \\
\hline n8 & 4 & 4 & $1 / 7$ & $1 / 2$ & $1 / 5$ & $1 / 2$ & $1 / 5$ & 1 & 3 & 4 & $1 / 6$ \\
\hline n9 & 3 & 6 & $1 / 7$ & $1 / 2$ & $1 / 5$ & $1 / 6$ & $1 / 5$ & $1 / 3$ & 1 & $1 / 6$ & $1 / 7$ \\
\hline$\eta 10$ & $1 / 6$ & 5 & $1 / 7$ & $1 / 2$ & $1 / 3$ & $1 / 4$ & $1 / 3$ & $1 / 4$ & 6 & 1 & $1 / 5$ \\
\hline n11 & $1 / 7$ & 6 & $1 / 7$ & 5 & 4 & 6 & 4 & 6 & 7 & 5 & 1 \\
\hline
\end{tabular}

Table - III (A) Sum Each Column of Reciprocal Values of Matrix - A

\begin{tabular}{|c|c|c|c|c|c|c|c|c|c|c|c|}
\hline $\mathbf{A}$ & $\eta 1$ & $\eta 2$ & $n 3$ & $\eta 4$ & n5 & n6 & n7 & $\eta 8$ & $\eta 9$ & $\eta 10$ & $\eta 11$ \\
\hline$\eta 1$ & 1.000 & 3.000 & 0.143 & .333 & 0.250 & 0.333 & 0.143 & 0.250 & 0.333 & 6.000 & 7.000 \\
\hline$\eta 2$ & 0.333 & 1.000 & 0.125 & 0.167 & 0.200 & 0.333 & 0.333 & 0.250 & 0.167 & 0.200 & 0.167 \\
\hline n3 & 7.000 & 8.000 & 1.000 & 7.000 & 6.000 & 7.000 & 5.000 & 7.000 & 7.000 & 7.000 & 7.000 \\
\hline$\eta 4$ & 3.000 & 6.000 & 0.143 & 1.000 & 3.000 & 2.000 & 0.333 & 2.000 & 2.000 & 2.000 & 0.200 \\
\hline n5 & 0.200 & 5.000 & 0.167 & 0.333 & 1.000 & 4.000 & 0.500 & 5.000 & 5.000 & 3.000 & 0.250 \\
\hline n6 & 3.000 & 3.000 & 0.143 & 0.500 & 0.250 & 1.000 & 0.333 & 2.000 & 6.000 & 4.000 & 0.167 \\
\hline n7 & 7.000 & 3.000 & 0.200 & 3.000 & 2.000 & 3.000 & 1.000 & 5.000 & 5.000 & 3.000 & 0.250 \\
\hline$\eta 8$ & 4.000 & 4.000 & 0.143 & 0.500 & 0.200 & 0.500 & 0.200 & 1.000 & 3.000 & 4.000 & 0.167 \\
\hline n9 & 3.000 & 6.000 & 0.143 & 0.500 & 0.200 & 0.167 & 0.200 & 0.333 & 1.000 & 0.167 & 0.143 \\
\hline n10 & 0.167 & 5.000 & 0.143 & 0.500 & 0.333 & 0.250 & 0.333 & 0.250 & 6.000 & 1.000 & 0.200 \\
\hline$\eta 11$ & 0.143 & 6.000 & 0.143 & 5.000 & 4.000 & 6.000 & 4.000 & 6.000 & 7.000 & 5.000 & 1.000 \\
\hline SUM & 28.843 & 50.000 & 2.492 & 18.833 & 17.433 & 24.583 & 12.376 & 29.083 & 42.500 & 35.367 & 16.543 \\
\hline
\end{tabular}

Table - III (B)

Normalized Relative Weight Of Matrix - A

\begin{tabular}{|c|c|c|c|c|c|c|c|c|c|c|c|}
\hline $\mathbf{A}$ & $\eta \mathbf{1}$ & $\eta 2$ & n3 & $\eta 4$ & n5 & n6 & n7 & n8 & n9 & $\eta \mathbf{1 0}$ & n11 \\
\hline$\eta 1$ & 0.035 & 0.060 & 0.057 & 0.018 & 0.014 & 0.014 & 0.012 & 0.009 & 0.008 & 0.170 & 0.423 \\
\hline$\eta 2$ & 0.012 & 0.020 & 0.050 & 0.009 & 0.011 & 0.014 & 0.027 & 0.009 & 0.004 & 0.006 & 0.010 \\
\hline n3 & 0.243 & 0.160 & 0.401 & 0.372 & 0.344 & 0.285 & 0.404 & 0.241 & 0.165 & 0.198 & 0.423 \\
\hline
\end{tabular}




\begin{tabular}{|l|l|l|l|l|l|l|l|l|l|l|l|}
\hline $\mathbf{\eta}$ & 0.104 & 0.120 & 0.057 & 0.053 & 0.172 & 0.081 & 0.027 & 0.069 & 0.047 & 0.057 & 0.012 \\
\hline $\mathbf{\eta 5}$ & 0.007 & 0.100 & 0.067 & 0.018 & 0.057 & 0.163 & 0.040 & 0.172 & 0.118 & 0.085 & 0.015 \\
\hline $\mathbf{\eta 6}$ & 0.104 & 0.060 & 0.057 & 0.027 & 0.014 & 0.041 & 0.027 & 0.069 & 0.141 & 0.113 & 0.010 \\
\hline $\mathbf{\eta 7}$ & 0.243 & 0.060 & 0.080 & 0.159 & 0.115 & 0.122 & 0.081 & 0.172 & 0.118 & 0.085 & 0.015 \\
\hline $\mathbf{\eta 8}$ & 0.139 & 0.080 & 0.057 & 0.027 & 0.011 & 0.020 & 0.016 & 0.034 & 0.071 & 0.113 & 0.010 \\
\hline $\boldsymbol{\eta 9}$ & 0.104 & 0.120 & 0.057 & 0.027 & 0.011 & 0.007 & 0.016 & 0.011 & 0.024 & 0.005 & 0.009 \\
\hline $\boldsymbol{\eta 1 0}$ & 0.006 & 0.100 & 0.057 & 0.027 & 0.019 & 0.010 & 0.027 & 0.009 & 0.141 & 0.028 & 0.012 \\
\hline $\mathbf{\eta 1 1}$ & 0.005 & 0.120 & 0.057 & 0.265 & 0.229 & 0.244 & 0.323 & 0.206 & 0.165 & 0.141 & 0.060 \\
\hline SUM & $\mathbf{1 . 0 0 0}$ & $\mathbf{1 . 0 0 0}$ & $\mathbf{1 . 0 0 0}$ & $\mathbf{1 . 0 0 0}$ & $\mathbf{1 . 0 0 0}$ & $\mathbf{1 . 0 0 0}$ & $\mathbf{1 . 0 0 0}$ & $\mathbf{1 . 0 0 0}$ & $\mathbf{1 . 0 0 0}$ & $\mathbf{1 . 0 0 0}$ & $\mathbf{1 . 0 0 0}$ \\
\hline
\end{tabular}

Table - III (C) Normalized Priority / Weight Value of Matrix - A

\begin{tabular}{|c|c|c|c|c|c|c|c|c|c|c|c|c|}
\hline $\mathbf{A}$ & $\eta \mathbf{1}$ & $\eta 2$ & n3 & $\eta 4$ & n5 & n6 & n7 & n8 & n9 & $\eta \mathbf{1 0}$ & $\eta \mathbf{1 1}$ & $\begin{array}{l}\text { Priority / } \\
\text { Weight }\end{array}$ \\
\hline$\eta 1$ & 0.035 & 0.060 & 0.057 & 0.018 & 0.014 & 0.014 & 0.012 & 0.009 & 0.008 & 0.170 & 0.423 & 0.074 \\
\hline$\eta 2$ & 0.012 & 0.020 & 0.050 & 0.009 & 0.011 & 0.014 & 0.027 & 0.009 & 0.004 & 0.006 & 0.010 & 0.016 \\
\hline n3 & 0.243 & 0.160 & 0.401 & 0.372 & 0.344 & 0.285 & 0.404 & 0.241 & 0.165 & 0.198 & 0.423 & 0.294 \\
\hline$\eta 4$ & 0.104 & 0.120 & 0.057 & 0.053 & 0.172 & 0.081 & 0.027 & 0.069 & 0.047 & 0.057 & 0.012 & 0.073 \\
\hline n5 & 0.007 & 0.100 & 0.067 & 0.018 & 0.057 & 0.163 & 0.040 & 0.172 & 0.118 & 0.085 & 0.015 & 0.077 \\
\hline n6 & 0.104 & 0.060 & 0.057 & 0.027 & 0.014 & 0.041 & 0.027 & 0.069 & 0.141 & 0.113 & 0.010 & $\mathbf{0 . 0 6 0}$ \\
\hline$\eta 7$ & 0.243 & 0.060 & 0.080 & 0.159 & 0.115 & 0.122 & 0.081 & 0.172 & 0.118 & 0.085 & 0.015 & 0.114 \\
\hline$\eta 8$ & 0.139 & 0.080 & 0.057 & 0.027 & 0.011 & 0.020 & 0.016 & 0.034 & 0.071 & 0.113 & 0.010 & $\mathbf{0 . 0 5 3}$ \\
\hline$n 9$ & 0.104 & 0.120 & 0.057 & 0.027 & 0.011 & 0.007 & 0.016 & 0.011 & 0.024 & 0.005 & 0.009 & $\mathbf{0 . 0 3 6}$ \\
\hline$\eta 10$ & 0.006 & 0.100 & 0.057 & 0.027 & 0.019 & 0.010 & 0.027 & 0.009 & 0.141 & 0.028 & 0.012 & 0.040 \\
\hline$\eta 11$ & 0.005 & 0.120 & 0.057 & 0.265 & 0.229 & 0.244 & 0.323 & 0.206 & 0.165 & 0.141 & 0.060 & 0.165 \\
\hline SUM & 1.000 & 1.000 & 1.000 & 1.000 & 1.000 & 1.000 & 1.000 & 1.000 & 1.000 & 1.000 & 1.000 & 1.000 \\
\hline
\end{tabular}

Table - III (D) Priority / Weighted Sum of Matrix - A

\begin{tabular}{|l|l|l|l|l|l|l|l|l|l|l|l|l|}
\hline $\mathbf{A}$ & $\mathbf{\eta 1}$ & $\mathbf{\eta 2}$ & $\mathbf{\eta}$ & $\mathbf{\eta} \mathbf{n 5}$ & $\mathbf{\eta 5}$ & $\mathbf{\eta 6}$ & $\mathbf{\eta 7}$ & $\mathbf{\eta 8}$ & $\mathbf{\eta 9}$ & $\mathbf{\eta 1 0}$ & $\mathbf{\eta 1 1}$ & $\begin{array}{l}\text { Priority/ } \\
\text { Weighted } \\
\text { sum }\end{array}$ \\
\hline $\mathbf{\eta 1}$ & 0.074 & 0.047 & 0.042 & 0.024 & 0.019 & 0.020 & 0.016 & 0.013 & 0.012 & 0.238 & 1.138 & $\mathbf{0 . 1 4 9}$ \\
\hline $\mathbf{\eta 2}$ & 0.025 & 0.016 & 0.037 & 0.012 & 0.015 & 0.020 & 0.038 & 0.013 & 0.006 & 0.008 & 0.027 & $\mathbf{0 . 0 2 0}$ \\
\hline $\mathbf{\eta 3}$ & 0.521 & 0.124 & 0.294 & 0.509 & 0.459 & 0.422 & 0.568 & 0.368 & 0.249 & 0.277 & 1.138 & $\mathbf{0 . 4 4 8}$ \\
\hline $\mathbf{\eta} \mathbf{4}$ & 0.223 & 0.093 & 0.042 & 0.073 & 0.230 & 0.121 & 0.038 & 0.105 & 0.071 & 0.079 & 0.033 & $\mathbf{0 . 1 0 1}$ \\
\hline $\mathbf{\eta 5}$ & 0.015 & 0.078 & 0.049 & 0.024 & 0.077 & 0.241 & 0.057 & 0.263 & 0.178 & 0.119 & 0.041 & $\mathbf{0 . 1 0 4}$ \\
\hline $\mathbf{\eta 6}$ & 0.223 & 0.047 & 0.042 & 0.036 & 0.019 & 0.060 & 0.038 & 0.105 & 0.213 & 0.158 & 0.027 & $\mathbf{0 . 0 8 8}$ \\
\hline $\mathbf{\eta 7}$ & 0.521 & 0.047 & 0.059 & 0.218 & 0.153 & 0.181 & 0.114 & 0.263 & 0.178 & 0.119 & 0.041 & $\mathbf{0 . 1 7 2}$ \\
\hline $\mathbf{\eta 8}$ & 0.298 & 0.062 & 0.042 & 0.036 & 0.015 & 0.030 & 0.023 & 0.053 & 0.107 & 0.158 & 0.027 & $\mathbf{0 . 0 7 7}$ \\
\hline
\end{tabular}




\begin{tabular}{|l|l|l|l|l|l|l|l|l|l|l|l|l|}
\hline п9 & 0.223 & 0.093 & 0.042 & 0.036 & 0.015 & 0.010 & 0.023 & 0.018 & 0.036 & 0.007 & 0.023 & $\mathbf{0 . 0 4 8}$ \\
\hline п10 & 0.012 & 0.078 & 0.042 & 0.036 & 0.026 & 0.015 & 0.038 & 0.013 & 0.213 & 0.040 & 0.033 & $\mathbf{0 . 0 5 0}$ \\
\hline $\boldsymbol{\eta 1 1}$ & 0.011 & 0.093 & 0.042 & 0.364 & 0.306 & 0.362 & 0.454 & 0.316 & 0.249 & 0.198 & 0.163 & $\mathbf{0 . 2 3 2}$ \\
\hline
\end{tabular}

TABLE - III (E) Normalized Eigen Value Of Matrix - A

\begin{tabular}{|c|c|c|c|c|c|c|c|c|c|c|c|c|}
\hline $\mathbf{A}$ & $\eta \mathbf{1}$ & $\eta 2$ & $\eta 3$ & $\eta 4$ & n5 & n6 & n7 & n8 & $\eta 9$ & $\eta 10$ & $\eta 11$ & $\lambda \max$ \\
\hline$\eta 1$ & 0.074 & 0.047 & 0.042 & 0.024 & 0.019 & 0.020 & 0.016 & 0.013 & 0.012 & 0.238 & 1.138 & 11.000 \\
\hline$\eta 2$ & 0.025 & 0.016 & 0.037 & 0.012 & 0.015 & 0.020 & 0.038 & 0.013 & 0.006 & 0.008 & 0.027 & 11.000 \\
\hline$n 3$ & 0.521 & 0.124 & 0.294 & 0.509 & 0.459 & 0.422 & 0.568 & 0.368 & 0.249 & 0.277 & 1.138 & 11.000 \\
\hline$\eta 4$ & 0.223 & 0.093 & 0.042 & 0.073 & 0.230 & 0.121 & 0.038 & 0.105 & 0.071 & 0.079 & 0.033 & 11.000 \\
\hline n5 & 0.015 & 0.078 & 0.049 & 0.024 & 0.077 & 0.241 & 0.057 & 0.263 & 0.178 & 0.119 & 0.041 & 11.000 \\
\hline n6 & 0.223 & 0.047 & 0.042 & 0.036 & 0.019 & 0.060 & 0.038 & 0.105 & 0.213 & 0.158 & 0.027 & 11.000 \\
\hline$\eta 7$ & 0.521 & 0.047 & 0.059 & 0.218 & 0.153 & 0.181 & 0.114 & 0.263 & 0.178 & 0.119 & 0.041 & 11.000 \\
\hline$\eta 8$ & 0.298 & 0.062 & 0.042 & 0.036 & 0.015 & 0.030 & 0.023 & 0.053 & 0.107 & 0.158 & 0.027 & 11.000 \\
\hline n9 & 0.223 & 0.093 & 0.042 & 0.036 & 0.015 & 0.010 & 0.023 & 0.018 & 0.036 & 0.007 & 0.023 & 11.000 \\
\hline n10 & 0.012 & 0.078 & 0.042 & 0.036 & 0.026 & 0.015 & 0.038 & 0.013 & 0.213 & 0.040 & 0.033 & 11.000 \\
\hline$\eta 11$ & 0.011 & 0.093 & 0.042 & 0.364 & 0.306 & 0.362 & 0.454 & 0.316 & 0.249 & 0.198 & 0.163 & 11.000 \\
\hline
\end{tabular}

After computing,

$\lambda \max =11.00, \mathrm{RI}=1.51, \mathrm{CR}=0.00<0.1$,

$\mathrm{w}=(0.0744,0.0155,0.2941,0.0727,0.0765,0.0603,0.1136,0.0526,0.0355,0.0396$, $0.1625)$.

Table - IV Evaluation Matrix Of All Criteria $(\eta 1, \eta 2 \ldots, \eta 11)$

\begin{tabular}{|l|l|l|l|l|l|l|l|l|l|l|l|}
\hline $\mathbf{B}$ & $\mathbf{\eta} \mathbf{1}$ & $\mathbf{\eta}$ & $\mathbf{\eta} 3$ & $\mathbf{\eta} 4$ & $\mathbf{\eta} 5$ & $\mathbf{\eta 6}$ & $\mathbf{\eta} \mathbf{7}$ & $\mathbf{\eta} \mathbf{8}$ & $\mathbf{\eta 9}$ & $\mathbf{\eta 1 0}$ & $\boldsymbol{\eta 1 1}$ \\
\hline $\mathbf{V}_{\mathbf{A}}$ & 1 & 3 & 7 & 6 & 8 & 7 & 5 & 6 & 7 & 9 & 8 \\
\hline $\mathbf{V}_{\mathbf{B}}$ & 10 & 4 & 6 & 5 & 7 & 6 & 6 & 5 & 8 & 8 & 8 \\
\hline $\mathbf{V}_{\mathbf{C}}$ & 5 & 3 & 5 & 4 & 4 & 6 & 6 & 8 & 7 & 7 & 6 \\
\hline $\mathbf{V}_{\mathbf{D}}$ & 8 & 4 & 3 & 3 & 4 & 5 & 5 & 6 & 7 & 7 & 8 \\
\hline $\mathbf{V}_{\mathbf{E}}$ & 7 & 3 & 5 & 4 & 3 & 5 & 3 & 4 & 3 & 5 & 6 \\
\hline
\end{tabular}

Table - IV (A) Evaluation Matrix Of All Criteria ( $\left.11, \eta^{2} \ldots . . n 11\right)$

\begin{tabular}{|c|c|c|c|c|c|c|c|c|c|c|c|}
\hline B & $\eta \mathbf{1}$ & $\eta^{2}$ & $\eta 3$ & $\eta 4$ & $\eta 5$ & n6 & n7 & $\eta 8$ & $\eta 9$ & $\eta \mathbf{1 0}$ & $\eta 11$ \\
\hline $\mathbf{V}_{\mathbf{A}}$ & 5.0 & 1.0 & 0.4 & 0.5 & 0.4 & 0.7 & 0.6 & 0.7 & 0.4 & 0.6 & 0.8 \\
\hline $\mathbf{V}_{\text {в }}$ & 0.5 & 0.8 & 0.5 & 0.6 & 0.4 & 0.8 & 0.5 & 0.8 & 0.4 & 0.6 & 0.8 \\
\hline $\mathbf{V}_{\mathbf{C}}$ & 1.0 & 1.0 & 0.6 & 0.8 & 0.8 & 0.8 & 0.5 & 0.5 & 0.4 & 0.7 & 1.0 \\
\hline $\mathbf{V}_{\mathbf{D}}$ & 0.6 & 0.8 & 1.0 & 1.0 & 0.8 & 1.0 & 0.6 & 0.7 & 0.4 & 0.7 & 0.8 \\
\hline$\overline{V_{E}}$ & 0.7 & 1.0 & 0.6 & 0.8 & 1.0 & 1.0 & 1.0 & 1.0 & 1.0 & 1.0 & 1.0 \\
\hline
\end{tabular}




\subsection{Results and Discussions}

From table V, Integrated weight can be calculated, and choose the ideal vendor by analyzing the findings of matrix B above.

Table $-\mathrm{V}$ Integrated Weight Matrix

\begin{tabular}{|c|c|c|c|c|c|c|c|c|c|c|c|c|c|}
\hline $\mathbf{C}$ & $\eta 1$ & $\eta 2$ & n3 & $n 4$ & n5 & n6 & $n 7$ & n8 & n9 & $\eta 10$ & n11 & $\mathbf{W}$ & \\
\hline $\mathbf{A}$ & 0.0744 & 0.0155 & 0.2941 & 0.0727 & 0.0765 & 0.0603 & 0.1136 & 0.0526 & 0.0355 & 0.0396 & 0.1625 & & \\
\hline $\mathbf{V}_{\mathbf{A}}$ & 1 & 3 & 7 & 6 & 8 & 7 & 5 & 6 & 7 & 9 & 8 & 0.884 & 1 \\
\hline $\mathbf{V}_{\mathbf{B}}$ & 10 & 4 & 6 & 5 & 7 & 6 & 6 & 5 & 8 & 8 & 8 & 0.581 & 5 \\
\hline $\mathbf{V}_{\mathrm{C}}$ & 5 & 3 & 5 & 4 & 4 & 6 & 6 & 8 & 7 & 7 & 6 & 0.718 & 4 \\
\hline $\mathbf{V}_{D}$ & 8 & 4 & 3 & 3 & 4 & 5 & 5 & 6 & 7 & 7 & 8 & 0.811 & 3 \\
\hline $\mathbf{V}_{\mathbf{E}}$ & 7 & 3 & 5 & 4 & 3 & 5 & 3 & 4 & 3 & 5 & 6 & 0.840 & 2 \\
\hline
\end{tabular}

Vendor A has a weight of 0.884 , Vendor B has a weight of 0.581 , Vendor $\mathrm{C}$ has a weight of 0.718 , Vendor D has a weight of 0.811 , and Vendor $E$ has a weight of 0.840 , as shown in Table V. As a result, we choose Vendor A as our IT outsourcing provider.

\section{Conclusions And Future Research}

This study shows how to use a decision model and the multi-criteria method to assist in the selection of an IT outsourcing firm. AHP presents extensive methodologies for selecting potential suppliers and evaluating the relationships between factors in this paper. The AHP method can be used to choose IT outsourcing projects with a variety of competing performance objectives. Managers can use the proposed decision model to quickly choose and analyze elements and features. AHP takes a quantitative and rigorous approach to outsourcing, helping managers to make well-informed decisions and achieve excellent results. Although the multi-criteria analysis is an excellent tool for answering the IT outsourcing question, the AHP technique is clearly applicable to other outsourcing challenges such as decision-making, investment project selection, and so on. The AHP method of calculating weights is considered subjective and random. Weights will be created by combining objectivity and subjectivity in a way that may be significantly superior to those created just because of subjectivity. This is one of the upcoming research areas we will be pursuing.

\section{Acknowledgement}

The authors would like to thank the management and principal, PSG College of Technology for generous support to carry out the research paper.

\section{References}

[1] Yang and J. Huang, "A decision model for IS outsourcing," International Journal of Information Management, vol. 20, pp. 225-39, 2000.

[2] K. S. Bhutta and F. Huq, "Supplier selection problem: a comparison of the total cost of ownership and analytical hierarchy process," Supply Chain Management, vol. 7, pp. 126 - 35, 2002. 
[3] R. M. Monckza and S. J. Trecha, "Cost-based supplier performance evaluation," Journal of Purchasing and Materials Management, vol. 24, pp. 2-7, 1988.

[4] A. M. Porter, "Tying down total cost," Purchasing, vol. 21, pp. 38-43, 1993.

[5] H. Min, "International supplier selection: a multi-attribute utility approach," International Journal of Physical Distribution and Logistics Management, vol. 24, pp. 24-33, 1994.

[6] C. A. Weber and J. R.Current, "A multiobjective approach to supplier selection," European Journal of Operational Research, vol. 68, pp. 173-84, 1993.

[7] K. Karpak and R. R. Kasuganti, "An application of visual interactive goal programming: acase in supplier selection decisions," Journal of Multi-criterion Decision Making, vol. 8, pp. 93-105, 1999 ,

[8] S.H. Ghodsypour and C. O’Brien, "A decision support system for supplier selection using an integrated analytic hierarchy process and linear programming," International Journal of Production Economics, vol. 56, pp. 199-212, 1998.

[9] F. Liu, F. Y. Ding and V. Lall, "Using data envelopment analysis to compare suppliers for supplier selection and performance improvement," Supply Chain Management: An International Journal, vol.5, pp. 143-50, 2000.

[10] T. L. Saaty, “The analytic hierarchy process,” New York, McGraw-Hil, 1980.

[11] M. J. Schniederjans and T. Garvin, "Using the analytic hierarchy process and multi-objective programming for the selection of cost drivers in activity based costing," European Journal of Operational Research, vol. 100, pp. 72-80, 1997.

[12] G. W. Dickson, "An analysis of vendor selection systems and decisions," Journal of Purchasing, vol. 2, pp. 5-17, 1966.

[13] C. A. Weber, J. R. Current and W. C. Benton, "Vendor selection criteria and methods," European Journal of Operational Research, vol. 50, pp. 2-18, 1991.

[14] K. L. Choy, W. B. Lee, and L. C. Choy, "A knowledge-based supplier intelligence retrieval system for outsource manufacturing," Knowledge- Based Systems, vol. 18, pp. 1-17, 2005.

[15] A. Mandal and S. G. Deskmukh, "Vendor selection using interpretive structural modeling (ISM)," International Journal of Operations and Production Management, vol. 14, pp. 52- 9, 1994.

[16] C. Macharis, J. Springael, K. De Brucher and A. Verbeke, "PROMETHEE and AHP: the design of operational synergies in multicriteria analysis. Strengthening PROMETHEE with ideas of AHP," European Journal of Operational Research, vol. 153, pp. 307-17, 2004.

[17] Prashaya Fusiripong, Fauziah Baharom and Yuhains Yusof, "Identification of Multi-criteria for supplier selection in IT project outsourcing", The 2nd International Conference on Applied Science and Technology 2017 (ICAST'17) AIP Conf. Proc.1891, 0200421-1- 020042-7.

[18] Adhikary, P., Roy, P. K., \& Mazumdar, A. (2015). Optimal renewable energy project selection: A multi-criteria optimization technique approach. Global Journal of Pure and Applied Mathematics, 11(5), 3319-3329.

[19] Alexandrova, M. (2012). It outsourcing partnerships: Empirical research on key success factors in bulgarian organization Management: Journal of ContemporaryManagement Issues, 17(2), 31-50.

[20] Bahli, B.,\&Rivard, S. (2013). Cost escalation in information technology outsourcing: A moderated mediation study. Decision Support Systems, 56, 37-47.

[21] Chang, S.-I., Yen, D. C., Ng, C. S.-P., \& Chang, W.-T. (2012). An analysis of it/is outsourcing provider selection for small- and medium-sized enterprises in taiwan. Information \& Management 49(5), 199 - 209. doi:http://dx.doi.org/10.1016/j.im.2012.03.001

[22] Dickson, G. W. (1996). An analysis of vendor selection systems and decisions.

[23] Digalwar, A. K., Borade, A., \& Metri, B. (2014). A fuzzy ahp approach for supplier selection. Operations and Supply Chain Management, 7(2), 46-53.

[24] Faisal, M. N., \& Raza, S. A. (2016). It outsourcing intent in academic institutions in gcc countries:An empirical investigation and multi-criteria decision model for vendor selection. Journal of Enterprise Information Management, 29(3), 432-453.

[25] Hermann, R., Baumgartner, R. J., Vorbach, S., Ragossnig, A., \& Pomberger, R. (2015). Evaluation and selection of decision-making methods to assess landfill mining projects. Waste Management \& Research, 33(9), 822- 832. 
[26] Ismail, F. F., \& Razali, R. (2014, 23-24 Sept. 2014). Contributing factors of successful software testing outsourcing. Paper presented at the Software Engineering Conference (MySEC), 2014 8th Malaysian.

[27] Karami, A., \& Guo, Z. (2012, 4-7 Jan. 2012). A fuzzy logic multi-criteria decision framework for selecting it service providers. Paper presented at the System Science (HICSS), 2012 45th Hawaii International Conference on.

[28] Khan, H., \& Faisal, M. N. (2015). A grey-based approach for erp vendor selection in small and medium enterprises in qatar. International Journal of Business Information Systems, 19(4), 465-487.

[29] Kronawitter, K., Wentzel, C., \& Papadaki, M. (2013). It application outsourcing in europe: Longterm outcomes, success factors and implications for ito maturity. Paper presented at the System Sciences (HICSS), 2013 46th Hawaii International Conference.

[30] Mukherjee, S., \& Mukherjee, K. (2015). Selection decisions of offshore into service providers for strategic alliances- an ahp-based approach. International Journal of Business Information Systems, 20(2), 238-269.

[31] Qiang, R., \& Li, D. (2015). An inhomogeneous multi-attribute decision making method and application to it/is outsourcing provider selection. International Journal of Industrial Engineering, 22(2).

[32] Rezaeisaray, M., Ebrahimnejad, S., \& Khalili-Damghani, K. (2016). A novel hybrid mcdm approach for outsourcing supplier selection: A case study in pipe and fittings manufacturing. Journal of Modelling in Management, 11(2), 536-559.

[33] Smuts, H., Kotz, P., Merwe, A. v. d., \& Loock, M. (2015, 14-15 Oct. 2015). Threats and opportunities for information systems outsourcing. Paper presented at the 2015 International Conference on Enterprise Systems (ES).

[34] Smuts, H., Merwe, A. v. d., Kotz, P., \& Loock, M. (2010). Critical success factors for information systems outsourcing management: A software development lifecycle view. Paper presented at the Proceedings of the 2010 Annual Research Conference of the South African Institute of Computer Scientists and Information Technologists, Bela Bela, South Africa.

[35] Thakur, V., \& Anbanandam, R. (2015). Supplier selection using grey theory: A case study from indian banking industry. Journal of Enterprise Information Management,28(6), 769- 787.

[36] Ullah Khan, S., Niazi, M., \& Ahmad, R. (2010). Critical success factors for offshore software development outsourcing vendors: An empirical study. In M. Ali Babar, M.Vierimaa, \& M. Oivo (Eds.), Product- focused software process improvement: 11th International conference, profes 2010, limerick, ireland, june 21-23, 2010. Proceedings (pp. 146-160). Berlin. 